\title{
Las Redes Sociales y su Influencia en la Salud Mental de los Estudiantes Universitarios: Una Revisión Sistemática
}

Jonathan Martínez-Líbano - Universidad SEK, Chile

Nicol González Campusano - Universidad de las Américas, Chile

Javiera Ignacia Pereira Castillo - Universidad de las Américas, Chile

iD 0000-0003-0774-2691

(D) $0000-0003-3521-1150$

(D) $0000-0001-8966-146 \mathrm{X}$

Recepción: 06.01.2022 | Aceptado: 10.01.2022

Correspondencia a través de ORCID: Jonathan Martínez-Líbano

0000-0003-0774-2691

Citar: Martinez-Libano, J, Gonzalez Campusano, N y Pereira Castillo, Jl (2022). Las Redes Sociales y su Influencia en la Salud Mental de los Estudiantes Universitarios: Una Revisión Sistemática. REIDOCREA, 11(4), 44-57.

Área o categoría del conocimiento: Educación Superior

Resumen: Los problemas de salud mental y su relación con las redes sociales son fenómenos relevantes de estudiar dada su alta incidencia y utilización entre los estudiantes universitarios. El objetivo de esta revisión sistemática fue determinar la influencia de las redes sociales en la salud mental de los estudiantes universitarios y establecer factores de riesgos y posibles impactos. La búsqueda se realizó siguiendo la metodología PRISMA en los buscadores Web of Science, Scopus, Pubmed, Taylor and Francis y Medline, entre enero de 2018 a diciembre de 2021, además se evalúo la calidad metodológica a través de la escala Newcastle-Ottawa (NOS). Respecto a los resultados al término de la búsqueda se lograron identificar trece estudios relevantes para analizarlos. Al concluir se logró estipular que los altos niveles de uso de las redes sociales en estudiantes universitarios se correlacionaron con la aparición de depresión, ansiedad, estrés, adicción a internet, Fomo, problemas de aprendizaje y trastornos del sueño. Estos resultados deben llamar la atención de autoridades académicas y políticas para prevenir la aparición de problemas de salud mental en estudiantes universitarios.

Palabra Clave: Salud Mental en Redes Sociales

Social Networks and Their Influences on the Mental Health of University Students: A Systematic Review

\begin{abstract}
Mental health problems and their relationship with social networks are relevant phenomena to study given their high incidence and use among university students. The objective of this systematic review was to determine the influence of social networks on the mental health of university students and to establish risk factors and possible impacts. The search was carried out following the PRISMA methodology in the Web of Science, Scopus, Pubmed, Taylor and Francis and Medline search engines, between January 2018 and December 2021, in addition, the methodological quality was evaluated through the Newcastle-Ottawa Scale (NOS). Regarding the results at the end of the search, thirteen relevant studies were identified for analysis. At the conclusion, it was possible to stipulate that the high levels of use of social networks in university students were correlated with the appearance of depression, anxiety, stress, internet addiction, Fomo, learning problems and sleep disorders. These results should attract the attention of academic and political authorities to prevent the appearance of mental health problems in university students.
\end{abstract}

Keywords: Mental Health in Social Networks

\section{INTRODUCCIÓN}

En la última década, el aumento de la conectividad en línea y el acceso a nuevas tecnologías como los teléfonos inteligentes han allanado el camino para que las redes sociales se conviertan en un método destacado para la interacción social y el acceso a la información en estudiantes alrededor de todo el mundo (Sadri et al., 2019; Sharif Hossen \& Aminul Islam, 2021). La multiplicación de los sitios de redes sociales ha llevado a una mayor frecuencia de uso entre los adultos jóvenes (Newman et al., 2021; 
Wojdan et al., 2021). Si bien la asociación con el bienestar mental sigue siendo controvertida, los altos niveles de uso de las redes sociales se correlacionaron con comportamientos problemáticos, baja autoestima y síntomas depresivos (El-Khoury et al., 2021).

La salud mental entre los estudiantes universitarios representa un importante y creciente problema de salud pública para el que se necesitan datos epidemiológicos (Duffy et al., 2019; Eisenberg et al., 2007). El ingreso a la educación superior es un momento de transición que coincide con la edad típica de aparición de una enfermedad mental grave (Farrell et al., 2017). En cuanto a la prevalencia de los problemas de salud mental en estudiantes de educación superior chilena, se ha encontrado que el $27 \%$ de los estudiantes presenta síntomas de depresión severa, un 10\% cumple con las características del trastorno bipolar, el $24 \%$ presenta consumo problemático de alcohol y un $15 \%$ sufre de trastornos alimenticios (Baader $M$ et al., 2014). En otras investigaciones se asegura que en Chile el $15 \%$ de los estudiantes universitarios presentan problemas de salud mental severo, la depresión moderada se incrementó desde $17.7 \%$ a $20.7 \%$, y la depresión severa en un $5.2 \%$, la ansiedad severa aumento desde un $16.7 \%$ a un $26.4 \%$, y el estrés severo han aumentado desde el $9.4 \%$ a un 15.9\% (Antúnez \& Vinet, 2013), finalmente la Red Universitaria G9 refiere que el $46 \%$ de los estudiantes encuestados presenta síntomas depresivos, el 46\% muestra ansiedad y el $54 \%$ padece estrés, mientras que el $29,7 \%$ sufre los tres problemas a la vez, estos niveles se pueden incrementar (Barrera-Herrera et al., 2019), más aún si en el periodo académico los estudiantes se ven afectados por una pandemia como la del COVID-19 (Martínez-Líbano, 2020; Martínez-Líbano \& Yeomans Cabrera, 2021). La pandemia ha reforzado la hipótesis de la existencia de un descenso en la calidad de vida y en la salud mental de las personas alrededor de todo el mundo y los estudiantes de entidades de educación superior no estarían fuera de dichas consecuencias (Kaparounakia Chrysi K. et al., 2020). La inestabilidad de la vida y los problemas de salud mental y física existentes, la incertidumbre sobre el futuro y pérdida de autonomía (Gruber et al., 2020), la transición repentina al aprendizaje en línea (Besser et al., 2020), los altos niveles de estrés y agotamiento (Humphrey, 2013), la sensación de tensión, miedo a la infección, insomnio y bajo estado de ánimo (Yang et al., 2020), el aumento de la angustia psicológica (Hamza et al., 2020), el aumento de la depresión moderada a grave y angustia emocional debido a COVID-19 (Xin et al., 2020), podrían llevar a los estudiantes universitarios a estar particularmente vulnerables y predispuestos a la a problemas de salud mental severos, creando de esta forma una crisis de salud pública (Tasnim et al., 2020). Lo anterior podría llevar los estudiantes universitarios experimentar estrés y burnout, el cual si se acompaña de una falta de actividad física y una dieta adecuada actuaran contra la salud y el bienestar psicológico de su alumnado (García-Ramírez et al., 2017).

Existen diversos estudios que muestran la relación entre el uso de las redes sociales y la salud mental (Aliverdi et al., 2021; Li et al., 2021; Maulik et al., 2010; Rusca et al., 2021), por ejemplo el uso de las redes sociales puede llevar a la aparición de la depresión (Kramer, 2021; Mason et al., 2009; Ragheb et al., 2021), en efecto el incremento de la depresión en la población juvenil está relacionada con el mal uso de las redes sociales, interfiriendo y afectando por tanto en aspectos importantes para un correcto desarrollo vital (Basterra Olives \& Cabrera Hernández, 2021). La ansiedad y las redes sociales también son fenómenos que han sido correlacionados (Smyth et al., 2015; Zhou et al., 2019), lo anterior podría deberse a la presentación de habilidades sociales deficitarias generando un uso excesivo de las redes sociales (Smyth et al., 2015), por lo tanto los estudiantes universitarios para cubrir la baja autoestima, compensar la carencia de habilidades sociales, y el aislamiento están usualmente conectados a las redes sociales, las cuales están relacionadas con la sintomatología 
depresiva (Padilla-Romero \& Ortega-Blas, 2017). La adicción a internet es otro tema que ha sido relacionado con problemas de salud mental en estudiantes de educación superior, sin embargo no está necesariamente relacionada con el tiempo pasado en internet, sino más bien con un patrón de uso desadaptativo (Moromizato et al., 2017), en la misma línea la adicción a los teléfonos inteligentes y la necesidad de estar conectados a internet en todo momento, es común entre las personas que sienten una necesidad urgente de mantenerse en contacto con los demás en todo momento, lo anterior sugiere que se debe concientizar sobre los efectos negativos de este uso excesivo de teléfonos inteligentes con relación a factores como; el sueño, la salud, la concentración y la comprensión; pero además hay consecuencias como la abstinencia, depresión y destrucción de las relaciones sociales. (Gao et al., 2018; Ivanova et al., 2020; James \& Drennan, 2005; Thomée et al., 2011)

En un estudio realizado en estudiantes universitarios, con una muestra total de 13.929 participantes, reveló una correlación positiva entre el uso problemático de Facebook y la angustia psicológica (Marino et al., 2018). Además, se demostró que hay una correlación negativa entre el uso problemático de Facebook y el bienestar (satisfacción con la vida, salud mental positiva), en otras palabras, el uso problemático de Facebook disminuye el bienestar en las personas, y que una disminución de este uso problemático crea un aumento en el bienestar (Brailovskaia et al., 2020; Villacampa et al., 2018). Dicha red social tiene más de mil millones de usuarios activos, por lo que cualquier influencia que pudiera tener en la salud mental podría transformarse en un problema de salud pública grave (Pantic, 2014). En Chile el $77 \%$ de la población tiene acceso a internet, de las cuales un $71 \%$ utiliza las redes sociales (Apablaza-Campos et al., 2018), lo que vuelve esto un foco relevante de estudio y posibles problemas de salud mental.

Actualmente las redes sociales están permanentemente influyendo la vida y actividad académica de los estudiantes universitarios (Gregory \& Rutledge, 2016), y se han logrado identificar fenómenos específicos como el síndrome denominado FOMO - Fear of missing out (Síndrome de miedo a perder contacto) (Abel \& Buff, 2016a; Przybylski et al., 2013), la cual es una aterradora ansiedad donde uno siente que se está perdiendo de algo absolutamente fantástico, aquella sensación de que otros están teniendo una experiencia maravillosa y gratificante de la que no formamos parte (Yana Salluca et al., 2019).

Consecuentemente y en relación con todo lo anterior planteado, es que el objetivo general de esta revisión fue determinar la influencia de las redes sociales en la salud mental de los estudiantes universitarios y como objetivos secundarios, establecer factores de riesgos y posibles impactos en la salud mental.

\section{MATERIALES Y MÉTODOS}

Se realizó una revisión sistemática, que identificó artículos publicados en las bases de datos Web of Science, Scopus, PubMed, Taylor and Francis y Medline entre enero de 2018 a octubre de 2021, escritos en inglés y español.

\section{Estrategia de búsqueda}

La búsqueda bibliográfica fue realizada de acuerdo con el protocolo de informes preferidos para revisiones sistemáticas y metaanálisis, PRISMA (Moher et al., 2009; Page et al., 2020; Urrútia \& Bonfill, 2013). Se realizaron búsquedas en los campos de búsqueda de título, resumen y palabras claves en cada una de las bases de datos. Se utilizaron las siguientes palabras claves combinadas con operadores booleanos (OR/AND): ([“Mental Health" OR "Emotional Health"] AND ["Social network" OR "Social 
Networks" OR "Social Networking"] AND [“University students" OR “College students”]). La estrategia de búsqueda de la selección de los estudios se encuentra en la Figura 1.

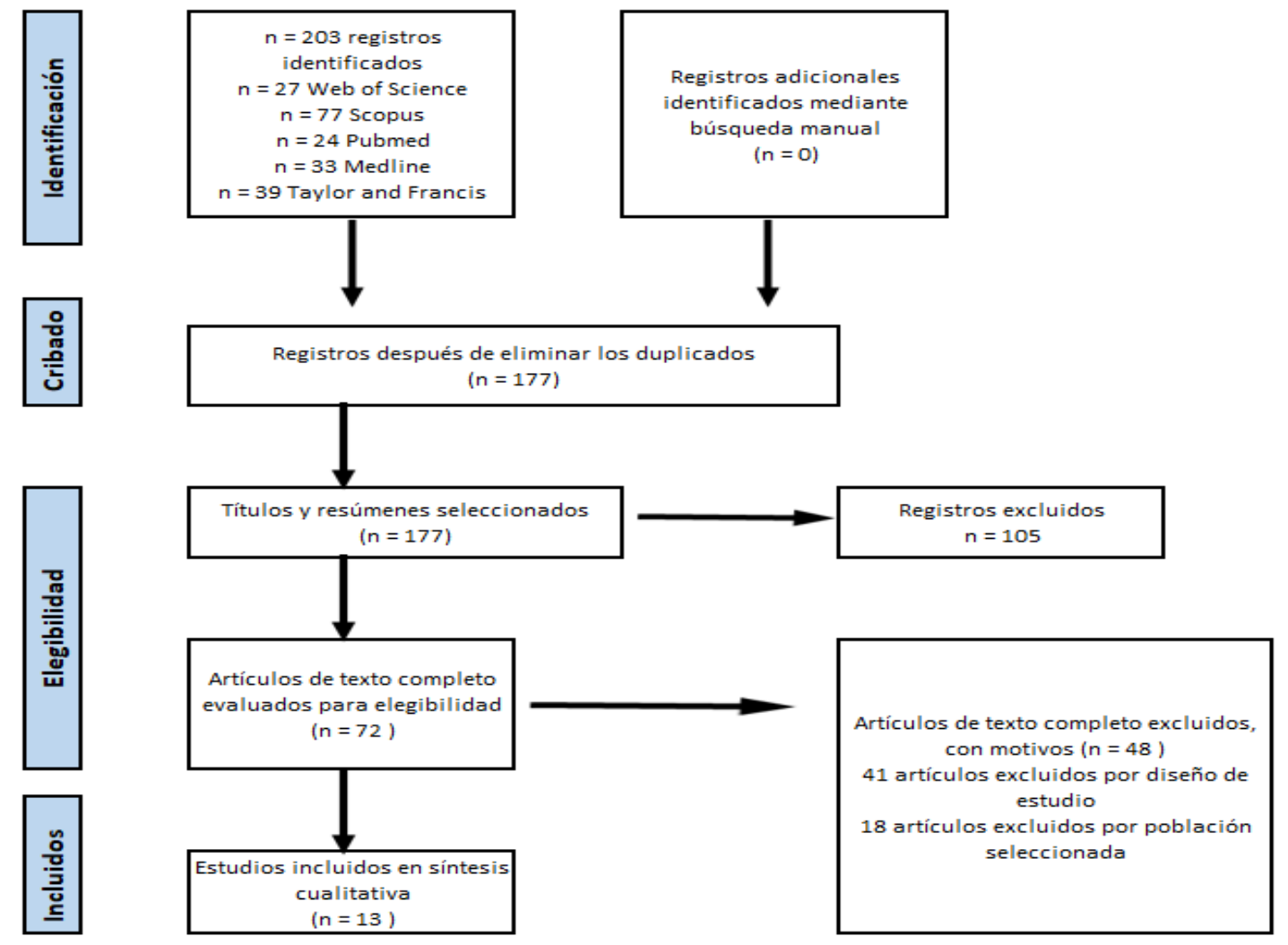

Figura 1. Diagrama de flujo PRISMA

\section{Criterios de selección y elegibilidad de estudios}

Se examinaron los títulos y resúmenes de cada artículo, luego se revisó el texto completo para determinar la elegibilidad. Los estudios fueron elegibles para su inclusión si: 1) siguieron un diseño de estudio transversal; 2) la población era en estudiantes universitarios; 3) con aplicación de instrumentos clínicos y no clínicos; 4) con resultados positivos y negativos; 5) textos en idioma inglés y español; 6) Si los datos de los artículos fueron durante la pandemia de COVID-19 y antes de esta. Se utilizaron los siguientes criterios de exclusión, 1) sujetos que no sean estudiantes universitarios; 2) que no tuviera la variable red social, plataformas digitales o uso de aparatos electrónicos inteligentes; 3) qué no considerarán la salud mental.

\section{Extracción de datos}

Se utilizó un formulario de extracción de datos para incluir datos relevantes: (1) Autor principal, (2) País, (3) Diseño del estudio, (4) Tamaño de la muestra, (5) Instrumento de medición, (6) Factores de riesgo asociados (7) impacto en la salud mental. 
Tabla 1. Resumen de las características de estudios

\begin{tabular}{|c|c|c|c|c|c|c|}
\hline $\begin{array}{l}\text { Autor } \\
\text { Principal }\end{array}$ & País & $\begin{array}{l}\text { Diseño } \\
\text { Estudio }\end{array}$ & $\begin{array}{l}\mathrm{N} \\
\text { Estudiantes }\end{array}$ & $\begin{array}{l}\text { Instrumentos de } \\
\text { Medición }\end{array}$ & Factores de Riesgo & Impacto en la Salud Mental \\
\hline $\begin{array}{l}\text { El-Khoury et } \\
\text { al., } 2021\end{array}$ & EE. UU & Transversal & 68 & $\begin{array}{l}\text { Instrumento propio: } \\
\text { Desintoxicación de las } \\
\text { Redes Sociales }\end{array}$ & $\begin{array}{l}\text { Baja actividad física, bajo } \\
\text { rendimiento académico. } \\
\text { Riesgo de uso problemático } \\
\text { de RRSS. (sobre todo con } \\
\text { Instagram). }\end{array}$ & $\begin{array}{l}\text { Síntomas de abstinencia al } \\
\text { querer dejar las redes } \\
\text { sociales, vida académica y } \\
\text { profesional se ve interferencia } \\
\text { negativa por sobreutilización }\end{array}$ \\
\hline $\begin{array}{l}\text { M. Tateno et } \\
\text { al., } 2019\end{array}$ & Japón & Transversal & 478 & $\begin{array}{l}\text { IAT; SAS-SV; HQ-25; } \\
\text { TACS }\end{array}$ & $\begin{array}{l}\text { Exceso de juegos en línea } \\
\text { (hombres), alta utilización de } \\
\text { redes sociales (mujeres), } \\
\text { bajas habilidades sociales, } \\
\text { trastornos psiquiátricos, } \\
\text { impulsividad }\end{array}$ & $\begin{array}{l}\text { Adicción a Internet, } \\
\text { aislamiento social, hikikimori, } \\
\text { depresión de tipo moderno } \\
\text { (MTD), }\end{array}$ \\
\hline $\begin{array}{l}\text { Tateno et al., } \\
2018\end{array}$ & Japón & Transversal & 602 & SAS-SV/IAT & $\begin{array}{l}\text { Tener entre } 20 \text { - } 29 \text { años, } \\
\text { utilización de teléfonos } \\
\text { inteligentes, incluyen una } \\
\text { edad más joven y } \\
\text { trastornos comórbidos del } \\
\text { desarrollo }\end{array}$ & Sensibilidad al rechazo \\
\hline $\begin{array}{l}\text { X. Hou et al., } \\
2019\end{array}$ & China & Transversal & 641 & $\begin{array}{l}\text { PSS/CES-D/S- } \\
\text { ANSIEDAD/ CD-RISC } \\
\text { /FIQ }\end{array}$ & Uso problemático del RRSS & $\begin{array}{l}\text { Depresión, ansiedad, estrés } \\
\text { percibido }\end{array}$ \\
\hline $\begin{array}{l}\text { Saini et al., } \\
2020\end{array}$ & India & Transversal & 220 & IAT adaptado & $\begin{array}{l}\text { Estudiantes con acceso libre } \\
\text { a internet, preferencias de } \\
\text { comunicarse en línea, } \\
\text { aislamiento en social }\end{array}$ & $\begin{array}{l}\text { Ansiedad, estrés, síntomas } \\
\text { depresivos, miedo a } \\
\text { perderse, cogniciones } \\
\text { desadaptativas, angustia } \\
\text { psiquiátrica, adicción a } \\
\text { internet }\end{array}$ \\
\hline $\begin{array}{l}\text { Zhao et al., } \\
2020\end{array}$ & China & Transversal & 512 & $\begin{array}{l}\text { Social Media Use, } \\
\text { COVID-19 Stressor, } \\
\text { PANAS, STSS-SM; } \\
\text { PHQ-9; GAD-7 }\end{array}$ & $\begin{array}{l}\text { Alto uso de internet, COVID- } \\
19, \text { exposición a los medios. }\end{array}$ & $\begin{array}{l}\text { Peor salud mental, depresión, } \\
\text { ansiedad, estrés. }\end{array}$ \\
\hline $\begin{array}{l}\text { Kitazawa et } \\
\text { al., } 2019 \\
\end{array}$ & Japón & Transversal & 1.258 & JIAT/ IHS/PSQI & Deserción escolar & $\begin{array}{l}\text { Menos felicidad, problemas } \\
\text { del sueño }\end{array}$ \\
\hline $\begin{array}{l}\text { Leung et al., } \\
2021\end{array}$ & China & Transversal & 760 & $\begin{array}{l}\text { CES-D, RSES, Fear of } \\
\text { fear of missing out } \\
\text { (FoMO) scale }\end{array}$ & $\begin{array}{l}\text { Cantidad de horas en las } \\
\text { RRSS, cantidad de } \\
\text { seguidores }\end{array}$ & $\begin{array}{l}\text { Las redes sociales inciden } \\
\text { negativamente en la } \\
\text { depresión, la autoestima y el } \\
\text { síndrome FOMO }\end{array}$ \\
\hline $\begin{array}{l}\text { Kolhar et al., } \\
2021\end{array}$ & Arabia & Transversal & 300 & Instrumento propio. & Aislamiento social & $\begin{array}{l}\text { Baja atención, problemas del } \\
\text { sueño, depresión, ansiedad y } \\
\text { cambios de humor }\end{array}$ \\
\hline $\begin{array}{l}\text { Lauckner et } \\
\text { al., } 2020\end{array}$ & EE. UU & Transversal & 255 & PHQ-9 & Alto uso de internet & $\begin{array}{l}\text { Impacto de redes sociales en } \\
\text { depresión }\end{array}$ \\
\hline $\begin{array}{l}\text { Bisen y } \\
\text { Deshpande, } \\
2020 \\
\end{array}$ & India & Transversal & 1.600 & IAT, BDI, BAI & $\begin{array}{l}\text { Alto uso de internet, uso } \\
\text { problemático de los } \\
\text { celulares }\end{array}$ & $\begin{array}{l}\text { Ansiedad, la depresión y la } \\
\text { impulsividad }\end{array}$ \\
\hline $\begin{array}{l}\text { Rasmussen, } \\
\text { et al., } 2020\end{array}$ & EE.UU & Transversal & 546 & DERS, PSS. & $\begin{array}{l}\text { Alto uso de las redes } \\
\text { sociales }\end{array}$ & $\begin{array}{l}\text { Dificultades con la regulación } \\
\text { de las emociones y } \\
\text { estrés percibido }\end{array}$ \\
\hline $\begin{array}{l}\text { Balouch et } \\
\text { al., } 2019 \\
\end{array}$ & Pakistán & longitudinal & 212 & $\mathrm{PHQ}-9, \mathrm{FIQ}$ & Alto uso de redes sociales & $\begin{array}{l}\text { Aumento de síntomas } \\
\text { depresivos en usuarios }\end{array}$ \\
\hline
\end{tabular}

\section{Evaluación de la Calidad}

Para la evaluación de la calidad de los estudios se utilizó la Escala Newcastle-Ottawa (NOS) adaptada para estudios transversales (Epstein et al., 2018). La NOS evalúa la calidad a partir del contenido, diseño e interpretación con gran confiabilidad y validez por sobre otras escalas (Cascaes da Silva et al., 2013). La escala consta de tres dimensiones: selección, comparabilidad y resultado. Hay siete categorías las cuales evalúan la representatividad de la muestra, la justificación del tamaño de la muestra, la comparabilidad entre los encuestados y los no encuestados, las determinaciones de la exposición, la comparabilidad basada en el diseño o análisis del estudio, la evaluación del resultado y la idoneidad del análisis estadístico. Se puede otorgar un total de nueve estrellas si el estudio cumple con ciertos criterios, con un máximo de cuatro estrellas asignadas para la dimensión de selección, un máximo de dos estrellas asignadas para 
la dimensión de comparabilidad y un máximo de tres estrellas asignadas para la dimensión de resultados (Epstein et al., 2018).

\section{RESULTADOS}

\section{Resultados de la Búsqueda}

En total, se identificaron 203 publicaciones acerca de la temática de influencia de las redes sociales en la salud mental de estudiantes universitarios. De ellos, 26 se eliminaron por duplicados. Se excluyeron 105 artículos por lectura de título, abstract y 59 por lectura de texto completo. De esta manera, 13 artículos cumplieron con los criterios de inclusión fueron seleccionados para la revisión sistemática.

\section{Características del Estudio}

Las características del estudio y los hallazgos de esta revisión se resumen en la tabla 1. El tamaño de las muestras de los 13 artículos osciló entre 68 y 1.600 participantes, con un total de 7.452 estudiantes de educación superior. Todos los estudios tuvieron un diseño de estudio transversal. Los 13 estudios se realizaron en diversos países alrededor del mundo EE.UU. $(n=3)$, China $(n=3)$, Japón $(n=3)$, India $(n=2)$, Arabia $(n=1)$, Pakistán $(n=1)$.

\section{Evaluación de la calidad metodológica}

El resultado de la evaluación de la calidad metodológica de los estudios se presenta en la Tabla 2. La calidad general de los estudios incluidos fue moderada, con un total de 6 a 7 estrellas. Hubo 9 estudios con siete estrellas (Balouch et al., 2019; Bisen \& Deshpande, 2020; Hou et al., 2019; Kitazawa et al., 2019; Lauckner et al., 2020; Leung et al., 2021; Rasmussen et al., 2020; Saini et al., 2020; Tateno et al., 2019) tres estudios con 6 estrellas (Kolhar et al., 2021; Tateno et al., 2018; Zhao \& Zhou, 2020), y hubo 1 estudio con 5 estrellas (El-Khoury et al., 2021)

\begin{tabular}{|c|c|c|c|c|c|c|c|c|c|}
\hline \multirow[t]{2}{*}{ Autor } & \multirow{2}{*}{$\begin{array}{l}\text { Punt. } \\
\text { Total }\end{array}$} & \multicolumn{4}{|l|}{ Selección } & \multirow{2}{*}{$\begin{array}{l}\text { Comparabilidad } \\
\text { Diseño y } \\
\text { Análisis } \\
\end{array}$} & \multicolumn{2}{|l|}{ Resultado } & \multirow[t]{2}{*}{ Calidad } \\
\hline & & $\begin{array}{l}\text { Representatividad } \\
\text { de la Muestra }\end{array}$ & $\begin{array}{l}\text { Tamaño } \\
\text { Muestra } \\
\end{array}$ & $\begin{array}{l}\text { No } \\
\text { Encuestados }\end{array}$ & $\begin{array}{l}\text { Comprobación } \\
\text { de exposición }\end{array}$ & & $\begin{array}{l}\text { Evaluación } \\
\text { Resultados } \\
\end{array}$ & $\begin{array}{l}\text { Test } \\
\text { Estadístico }\end{array}$ & \\
\hline $\begin{array}{l}\text { El-Khoury et } \\
\text { al., } 2021\end{array}$ & 5 & 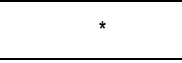 & * & & $*$ & & * & * & Satisfactorio \\
\hline $\begin{array}{l}\text { M. Tateno et } \\
\text { al., } 2019\end{array}$ & 7 & * & * & & ** & * & * & * & Bueno \\
\hline $\begin{array}{l}\text { Tateno et al., } \\
2018\end{array}$ & 6 & * & * & & $* *$ & & * & * & Satisfactorio \\
\hline $\begin{array}{l}\text { X. hou et al., } \\
2019\end{array}$ & 7 & * & * & & $* *$ & * & * & * & Bueno \\
\hline $\begin{array}{l}\text { Saini et al., } \\
2020\end{array}$ & 7 & * & * & * & * & * & * & * & Bueno \\
\hline $\begin{array}{l}\text { Zhao et al., } \\
2020\end{array}$ & 6 & * & * & & * & * & * & * & Satisfactorio \\
\hline $\begin{array}{l}\text { Kitazawa et al., } \\
2019\end{array}$ & 7 & * & * & * & $* *$ & * & * & * & Bueno \\
\hline $\begin{array}{l}\text { Leung et al., } \\
2021\end{array}$ & 7 & * & * & * & ** & & * & * & Bueno \\
\hline $\begin{array}{l}\text { Kolhar et al., } \\
2021\end{array}$ & 6 & * & * & * & * & & * & * & Satisfactorio \\
\hline $\begin{array}{l}\text { Lauckner et al., } \\
2020\end{array}$ & 7 & * & * & * & $* *$ & & * & * & Bueno \\
\hline $\begin{array}{l}\text { Bisen y } \\
\text { deshpande, } \\
2020 \\
\end{array}$ & 7 & * & * & * & $* *$ & & * & * & Bueno \\
\hline $\begin{array}{l}\text { Rassmussen et } \\
\text { al., } 2020\end{array}$ & 7 & * & * & * & $* *$ & & * & * & Bueno \\
\hline $\begin{array}{l}\text { Balouch et al., } \\
2019\end{array}$ & 7 & * & * & * & $* *$ & & * & * & Bueno \\
\hline
\end{tabular}




\section{Herramientas de medición}

Se utilizaron diversas escalas en los estudios tales como, IAT: Young's Internet Addiction Test (Young, 2016); SAS-Short Version: The Smartphone Addiction Scale (Kwon et al., 2014); (HQ-25): The 25-item Hikikomori Questionnaire (Teo et al., 2018) (52); TACS: Tarumi's modern-type depression trait scale Avoidance of social roles, Complaint and low Self-esteem (Kato et al., 2019); PSS: Perceived Stress Scale (Cohen, 1994); CES-D: Epidemiologic Studies Depression Scale (Radloff, 1977); S-Anxiety: the State Anxiety Subscale (Spielberger et al., 2015); CD-RISC: The Connor-Davidson Resilience Scale (Connor \& Davidson, 2003); FIQ: Facebook Intrusion Questionnaire (Elphinston \& Noller, 2011); Social Media Use (Lin et al., 2016); COVID-19 Stressor (Zhao \& Zhou, 2020); PANAS: Positive and Negative Affect Schedule (Watson et al., n.d.) (STSS-SM): Secondary Traumatic Stress Scale for Social Media Users (Mancini, 2019); PHQ-9: Patient Health Questionnaire depression (Kroenke et al., 2001); GAD-7: Generalized Anxiety Disorder Scale (Spitzer et al., 2006); (IHS): Interdependent Happiness Scale (Hitokoto \& Uchida, 2015); JIAT: Japanese version of internet addiction test (Osada, 2013) (PSQI): The Pittsburgh sleep quality index (Buysse et al., 1989); Fear of missing out (FoMO) scale (Abel \& Buff, 2016); RSES: Rosenberg Self-Esteem Scale (Rosenberg, 1965) Difficulties in Emotion Regulation Scale (DERS) (Gratz \& Roemer, 2004); BAl: Beck Anxiety Inventory (Beck et al., 1988); BDI: Beck Depression Inventory (Beck et al., 1961).

\section{Factores de riesgo asociados a estudiantes universitarios}

Dentro de los factores de riesgos para la influencia de las redes sociales sobre la salud mental de los estudiantes universitarios, encontramos: uso problemático de redes sociales $(n=8)$, trastornos psiquiátricos $(n=2)$, aislamiento social $(n=2)$, edad más joven $(n=2)$, uso problemático de los celulares $(n=2)$, baja actividad física $(n=1)$, bajo rendimiento académico $(n=1)$, exceso de juegos en línea (hombres) $(n=1)$, exceso utilización de redes sociales (mujeres) $(n=1)$, bajas habilidades sociales $(n=1)$, impulsividad $(n=1)$, utilización de teléfonos inteligentes $(n=1)$, estudiantes con acceso libre a internet $(n=1)$, preferencias de comunicarse en línea $(n=1)$, COVID-19 $(n=1)$, exposición a los medios $(n=1)$, deserción escolar $(n=1)$, cantidad de seguidores $(n=1)$.

\section{Impacto en la salud mental de los estudiantes universitarios}

Dentro de los impactos en la salud mental de los estudiantes universitarios debido al uso de las redes sociales e internet, encontramos: depresión $(n=8)$, ansiedad $(n=5)$, estrés $(n=4)$, adicción a Internet $(n=2)$, síndrome FOMO $(n=2)$, problemas del sueño $(n=2)$, síntomas de abstinencia $(n=1)$, vida académica y profesional se ve interferencia negativa por sobreutilización $(n=1)$, , aislamiento social $(n=1)$, hikikimori $(n=1)$, depresión de tipo moderno (MTD) $(n=1)$, sensibilidad al rechazo $(n=1)$, cogniciones desadaptativas $(n=1)$, angustia psiquiátrica $(n=1)$, peor salud mental $(n=1)$, menos felicidad $(n=1)$, baja autoestima $(n=1)$, baja concentración $(n=1)$, cambios de humor $(n=1)$, impulsividad $(n=1)$, dificultades con la regulación de las emociones $(n=1)$.

\section{DISCUSIÓN}

Las nuevas tecnologías y el acceso a Internet han cambiado drásticamente nuestra vida diaria y también ha alterado la forma en que nos comunicamos, esto se ve reflejando en una población tan sensible como son los estudiantes de educación superior. A medida que las aplicaciones de redes sociales se vuelven más populares, los usuarios están conectados más estrechamente a Internet y el tiempo que pasan con otras personas en el mundo real sigue disminuyendo (Tateno et al., 2018, 2019), una tendencia que sigue 
creciendo día a día entre los universitarios (González et al., 2009), este fenómeno se encuentra mayormente presente en las personas jóvenes, debido a que es en esta etapa cuando el grupo de pares adquiere mayor importancia y se hace más dependiente de las amistades como fuente de bienestar, y aunque en esta etapa continúa aumentando el nuevo sentido de individualidad, la autoimagen es muy dependiente de la opinión de terceros (Gaete, 2015). Por ende, las redes sociales al ser las fuentes de mayor comunicación e interacción con los otros, principalmente entre sujetos del mismo rango etario, por tanto, pasan a ser muy importantes para los adolescentes y su utilización es masiva (Boulianne \& Theocharis, 2020)

Esta revisión exploró el impacto en la salud mental de los estudiantes universitarios las redes sociales y el internet. Al término de esta revisión, se puede concluir que existe un uso problemático de internet y teléfonos en la última década entre los estudiantes universitarios, los resultados mostraron que en promedio presentarían un $47.4 \%$ de adicción a internet y teléfonos inteligentes (Carbonell, 2018; Qudah et al., 2019). Esto puede estar relacionado a diversos factores de riesgos como la baja autoestima, baja satisfacción en la vida, presentar sintomatología depresiva, ansiosa y bajo apoyo social percibido (Błachnio \& Przepiórka, 2018). El uso excesivo de las redes sociales tiene impacto en la salud mental en los universitarios, el $12,5 \%$ se enfrentan a problemas graves de salud mental (Bisen \& Deshpande, 2020). Los síntomas depresivos y la depresión fueron uno de los impactos psicológicos mayormente mencionados y citados en las investigaciones revisadas, lo anterior se puede deber a que las personas que reciben bajo apoyo social en la vida real, son propensas a aumentar el uso de redes sociales (Brailovskaia et al., 2018), así mismo las redes sociales pudieran generar problemas de regulación emocional (Rasmussen et al., 2020), al ver que otras personas tienen una vida más interesante que la nuestra, puede generar una dolorosa y profunda envidia a través de la comparación de las diversas realidades, haciendo que las personas presenten una bajos niveles de satisfacción con la vida (Balouch et al., 2019; Wang et al., 2019), repercutiendo negativamente en nuestra autoestima (Leung et al., 2021) y generando sentimientos de soledad y vacío (Zhang et al., 2020). La ansiedad puede estar asociada a la incapacidad de las personas de desconectarse de los medios digitales generando inclusive síntomas de abstinencia si es que no logran estar al tanto de lo que acontece en esta realidad virtual (El-Khoury et al., 2021), además ese miedo de perderse algo que está ocurriendo en las redes puede llevar al síndrome FOMO (Leung et al., 2021). El estrés también fue uno de los impactos psicológicos mayormente referidos, esto puede deberse a que cuando las personas están pasando por situaciones estresantes buscan apoyo emocional y social a través de las redes sociales y el teléfono inteligente es una forma rápida de poder buscar dicho apoyo, sin embargo, el buscar dicho apoyo y no encontrarlo también puede ser un foco de estrés (Dissing et al., 2019).

Existen diferencias entre hombres y mujeres en la utilización de internet, esto a razón que los hombres lo utilizan preferentemente para jugar juegos en línea, mientras que las mujeres se centran en redes sociales como facebook e instagram (Tateno et al., 2019), esta diferencia entre los géneros en el uso de las redes sociales se puede deber a que la corporalidad ha estado ligada irremisiblemente a lo social y a lo cultural (Moreno Vallejo, 2020), en nuestra sociedad occidental, la educación tiende a modelar nuestro cuerpo y a ajustarlo a las exigencias y normativas del entorno en que vivimos, adquiriendo el cuerpo una función muy relevante como mediador cultural (Behar A, 2010), lo que puede reflejarse en que se enseña desde niña el ensañamiento contra el propio cuerpo por no cumplir los estereotipos de belleza impuestos, los cuales son o llegan a ser muy ajenos a la realidad de miles de mujeres (Guzman Acuña \& Salazar Rocha, 2016). Actualmente está influencia en la importancia de la imagen corporal es transmitida por las redes sociales por su globalización y la importancia que tienen actualmente en la comunicación e interacción (Vergara, 2020). 
La pandemia de COVID 19 ha significado desafíos fundamentales para la forma en que entendemos nuestros nexos con otras personas (Oyanedel \& Paez, 2021) y ha mostrado una estrecha relación entre el empeoramiento de la salud mental y el uso de las redes sociales (Zhao \& Zhou, 2020), esto a razón de que las clases pasaron a ser en línea afectando todo el quehacer académico de estudiantes y docentes afectando sus actividades de aprendizaje (Kolhar et al., 2021; Yeomans \& Silva, 2020), además la clausura de lugares de trabajo y la cuarentena domiciliaria, ha ocasionado un salto en el uso por parte de la población de redes de telecomunicaciones para resolver temas de aprovisionamiento de bienes, conectividad social, y acceso a información (Agudelo et al., 2020). Existe una relación entre el empeoramiento de la salud mental y las redes sociales por la exposición a las noticias sobre desastres, y está a su vez se asocia con una mayor depresión para los participantes de la muestra con niveles altos del factor estresante (Hou et al., 2019), generando en los estudiantes de educación superior un gran agotamiento emocional (Martínez-Líbano et al., 2021), en este contexto la pandemia se encontró una relación entre las nuevas modalidades de aprendizaje a través de la tecnología, y el uso inadecuado de las redes sociales en los universitarios debido a la postergación de actividades y la baja autorregulación académica (RamírezGil et al., 2021).

Finalmente es fundamental seguir avanzando en estudiar los impactos de las redes sociales sobre la salud mental, así mismo en desarrollar instrumentos específicos o bien validar aquellos que se requieran para medir nuevos trastornos mentales que se pudieran estar desarrollando en la población de estudiantes universitarios.

\section{Fortalezas}

Este artículo de revisión sistemática examina y resume la literatura existente sobre la influencia de las redes sociales en la salud mental de los estudiantes universitarios. Se pudo reafirmar que, en la literatura actual, aun esta temática es muy controversial y no existen estudios que demuestran una relación positiva entre estos factores salud mental y las redes sociales. Sin embargo, hay factores de riesgo asociados que dan luces importantes sobre una posible repercusión del uso de estas plataformas digitales en la vida de los adultos jóvenes.

\section{Limitaciones}

Esta revisión sistemática tiene ciertas limitaciones dado que todos los estudios fueron transversales, lo que lleva a que en muchas investigaciones a que no se puede asumir la causalidad de las asociaciones. Además, en algunas solo se incluyó medidas de autoinforme e incluso cuestionarios realizados por los propios autores dejando de lado la validez y confiabilidad. Finalmente, muchos estudios dejan de lado variables que pueden moderar la relación entre el uso de las redes sociales en la salud mental, además, es necesario investigaciones experimentales para establecer relaciones de causalidad entre las redes sociales y la salud mental.

\section{Proyecciones}

Se espera con la revisión sistemática realizada, desarrollar un estudio de campo que permita ahondar la temática de las redes sociales en la salud mental en estudiantes universitarios en la población chilena, lo anterior se justifica dado que la mayoría de la investigación no se concentra en Latinoamérica y al realizar esta revisión se comprueba dicha situación al no existir investigaciones transversales en el periodo de selección realizado. 


\section{Referencias}

Abel, JP, \& Buff, CL (2016a). Social Media and the Fear of Missing Out: Scale Development and Assessment. In Journal of Business \& Economics Research-First Quarter, 14(1).

Abel, JP, \& Buff, CL (2016b). Social Media and the Fear of Missing Out: Scale Development and Assessment. In Journal of Business \& Economics Research-First Quarter, 14(1).

Agudelo, M, Chomali, E, Suniaga, J, Núñez, G, Jordán, J, Rojas, F, Negrete, J, Bravo, J, Bertolini, P, Katz, R, Callorda, F y Jung, J (2020). Las oportunidades de la digitalización en América Latina frente al Covid-19.

Aliverdi, F, Tourzani, ZM, Salehi, L, Qorbani, M, \& Mohamadi, F (2021). Social Networks and Internet Emotional Relationships on Mental Health and Quality of Life in Students: Structural Equation Modelling. https://doi.org/10.21203/rs.3.rs-1147915/v1

Antúnez, Z y Vinet, E (2013). Problemas de salud mental en estudiantes de una universidad regional chilena. Revista Médica de Chile, 141(2), 209-216.

Apablaza-Campos, A, Codina, L, Apablaza-Campos, A y Codina, L (2018). Social Media Live Streaming: estudio de caso y diseño de matriz de análisis. Cuadernos.Info, 43, 161-180. https://doi.org/10.7764/cdi.43.1342

Baader, T, Rojas, C, Molina, JL, Gotelli, M, Alamo, C, Fierro, C, Venezian, $S$ y Dittus, P (2014). Diagnóstico de la prevalencia de trastornos de la salud mental en estudiantes universitarios y los factores de riesgo emocionales asociados. Revista Chilena de Neuro-Psiquiatría, 52(3), 167-176. https://doi.org/10.4067/S071792272014000300004

Balouch, MA, Anwar, S, Ansari, MI, Rasheed, T, Ansari, MS, \& Balouch, N (2019). Impact of inline social networking on mental health among medical students of LUMHS Jamshoro, Sindh, Pakistan. Rawal Medical Journal, 44(3).

Barrera-Herrera, A, Neira-Cofré, M, Raipán-Gómez, P, RiquelmeLobos, P, \& Escobar, B (2019). Perceived social support and sociodemographic factors in relation to symptoms of anxiety, depression, and stress in Chilean university students. Revista de Psicopatologia y Psicologia Clinica, 24(2), 105-115. https://doi.org/10.5944/rppc.23676

Basterra Olives, M y Cabrera Hernández, L (2021). Las redes sociales y su influencia en trastornos como la depresión en los jóvenes. http://riull.ull.es/xmlui/handle/915/23966

Beck, AT, Epstein, N, Brown, G, \& Steer, RA (1988). An inventory for measuring clinical anxiety: Psychometric properties. Journal of Consulting and Clinical Psychology, 56(6), 893-897.

Beck, AT, Ward, CH, Mendelson, M, Mock, J, \& Erbaugh, J (1961). Archives of General Psychiatry, 4, 561-571.

Behar, R (2010). La construcción cultural del cuerpo: El paradigma de los trastornos de la conducta alimentaria. Revista Chilena de Neuro-Psiquiatría, 48(4), 319-334. https://doi.org/10.4067/S071792272010000500007

Besser, A, Flett, GL, \& Zeigler-Hill, V (2020). Adaptability to a sudden transition to online learning during the COVID-19 pandemic:
Understanding the challenges for students. Scholarship of Teachingand Learning in Psychology. Advance Online Publication. https://doi.org/10.1037/st10000198

Bisen, SS, \& Deshpande, Y (2020). Prevalence, predictors, psychological correlates of internet addiction among college students in India: A comprehensive study. Anadolu Psikiyatri Dergisi, 21(2), 117-123. https://doi.org/10.5455/apd.47328

Błachnio, A, \& Przepiórka, A (2018). Facebook intrusion, fear of missing out, narcissism, and life satisfaction: A cross-sectional study. Psychiatry Research, 259, 514-519. https://doi.org/10.1016/j.psychres.2017.11.012

Boulianne, S, \& Theocharis, Y (2020). Young People, Digital Media, and Engagement: A Meta-Analysis of Research. Social Science Computer Review, 38(2), 111-127. https://doi.org/10.1177/0894439318814190

Brailovskaia, J, Bierhoff, HW, Rohmann, E, Raeder, F, \& Margraf, J (2020). The relationship between narcissism, intensity of Facebook use, Facebook flow and Facebook addiction. Addictive Behaviors Reports, 11, 100265. https://doi.org/10.1016/j.abrep.2020.100265

Brailovskaia, J, Teismann, T, \& Margraf, J (2018). Physical activity mediates the association between daily stress and Facebook Addiction Disorder ( $F A D$ - A longitudinal approach among German students. Computers in Human Behavior, 86, 199-204. https://doi.org/10.1016/j.chb.2018.04.045

Buysse, DJ, Reynolds, CF, Monk, TH, Berman, SR, \& Kupfer, DJ (1989). The Pittsburgh sleep quality index: A new instrument for psychiatric practice and research. Psychiatry Research, 28(2), 193213. https://doi.org/10.1016/0165-1781(89)90047-4

Carbonell, X (2018). La Supuesta Adicción a las Redes Sociales. In Estudos sobre as Dependências: Contributos para a Prática (pp. 219-238). Axioma - Publicações da Faculdade de Filosofia. https://doi.org/10.17990/axi/2018_9789726973027_219

Cascaes da Silva, F, Beatriz Angélica Valdivia Arancibia, T, da Rosa lop, R, Jose Barbosa Gutierres Filho, P, \& da Silva, R (2013). Escalas y listas de evaluación de la calidad de estudios científicos. Revista Cubana de Información En Ciencias de La Salud (ACIMED), 24(3), 295-312.

Cohen, S (1994). Perceiver Stress Scale. www.mindgarden.com

Connor, KM, \& Davidson, JRT (2003). Development of a new resilience scale: The Connor-Davidson Resilience Scale (CDRISC). Depression and Anxiety, 18(2), 76-82. https://doi.org/https://doi.org/10.1002/da.10113

Dissing, AS, Jørgensen, TB, Gerds, TA, Rod, NH, \& Lund, R (2019). High perceived stress and social interaction behaviour among young adults. A study based on objective measures of face-to-face and smartphone interactions. PLoS ONE, 14(7). https://doi.org/10.1371/journal.pone.0218429

Duffy, A, Saunders, KEA, Malhi, GS, Patten, S, Cipriani, A, McNevin, SH, MacDonald, E, \& Geddes, J (2019). Mental health care for university students: a way forward? The Lancet Psychiatry, 6(11), 885-887. https://doi.org/10.1016/S2215-0366(19)30275-5 
Eisenberg, D, Gollust, SE, Golberstein, E, \& Hefner, JL (2007). Prevalence and Correlates of Depression, Anxiety, and Suicidality Among University Students. 77(4), 534-542. https://doi.org/10.1037/0002-9432.77.4.534

El-Khoury, J, Haidar, R, Kanj, RR, Bou Ali, L, \& Majari, G (2021). Characteristics of social media 'detoxification' in university students. Libyan Journal of Medicine, 16(1). https://doi.org/10.1080/19932820.2020.1846861

Elphinston, RA, \& Noller, P (2011). Time to Face It! Facebook Intrusion and the Implications for Romantic Jealousy and Relationship Satisfaction. Cyberpsychology, Behavior, and Social Networking, 14(11), 631-635. https://doi.org/10.1089/cyber.2010.0318

Epstein, S, Roberts, E, Sedgwick, R, Finning, K, Ford, T, Dutta, R, \& Downs, J (2018). Poor school attendance and exclusion: A systematic review protocol on educational risk factors for self-harm and suicidal behaviours. BMJ Open, 8(12), 1-5. https://doi.org/10.1136/bmjopen-2018-023953

Farrell, S, Kapur, N, While, D, Appleby, L, \& Windfuhr, K (2017). Suicide in a national student mental health patient population, 1997 2012. Crisis, 38(2), 82-88. https://doi.org/10.1027/02275910/a000412

Gaete, V (2015). Adolescent psychosocial development. In Revista Chilena de Pediatria, 86(6), 436-443. Sociedad Chilena de Pediatria. https://doi.org/10.1016/j.rchipe.2015.07.005

Gao, T, Li, J, Zhang, H, Gao, J, Kong, Y, Hu, Y, \& Mei, S (2018). The influence of alexithymia on mobile phone addiction: The role of depression, anxiety and stress. Journal of Affective Disorders, 225, 761-766. https://doi.org/10.1016/j.jad.2017.08.020

García-Ramírez, JM, Álvarez Castro, I, Campos Rodríguez, LM, Fernández García, T, García McMullin, SC, Ibáñez Praena, A, López Márquez, M, Ortega Castro, A y Varón Povedano, C (2017). Validación del cuestionario de Salud Eduactiva. ReiDoCrea. https://doi.org/10.30827/Digibug.45030

González, AL, Herrero García, N, González, AL y Herrero García, N. (2009). Universidad y sociedad. In Revista Universidad y Sociedad, 11(5).

Gratz, KL, \& Roemer, L (2004). Multidimensional Assessment of Emotion Regulation and Dysregulation: Development, Factor Structure, and Initial Validation of the Difficulties in Emotion Regulation Scale. Journal of Psychopathology and Behavioral Assessment, 26(1), 41-54. https://doi.org/10.1023/B:JOBA.0000007455.08539.94

Gregory, EM, \& Rutledge, PB (2016). Exploring Positive Psychology: The Science of Happiness and Well-Being: The Science of Happiness and Well-Being. ABC-CLIO.

Gruber, J, Prinstein, MJ, Clark, LA, Rottenberg, J, Abramowitz, JS, Albano, AM, Aldao, A, Borelli, JL, Chung, T, Davila, J, Forbes, EE, Gee, DG, Hall, GCN, Hallion, LS, Hinshaw, SP, Hofmann, SG, Hollon, SD, Joormann, J, Kazdin, AE, ... Weinstock, LM (2020). Mental Health and Clinical Psychological Science in the Time of COVID-19: Challenges, Opportunities, and a Call to Action. American Psychologist. https://doi.org/10.1037/amp0000707

Guzman Acuña, J, \& Salazar Rocha, I (2016). Sociocultural pressure to wards body image of women and its effects in their academic performance. Revista Internacional de Ciencias Sociales y Humanidades, SOCIOTAM, XXVI, 11-41.
Hamza, A, Saleem, A, Noori, A, \& Thaaer, H (2020). The Psychological, Mental and Physical Effects of Defamation Crimes and Their Severity on the Health of the Iraqi Individual. Indian Journal of Forensic Medicine \& Toxicology, 14(2), 862-867.

Hitokoto, H, \& Uchida, Y (2015). Interdependent Happiness: Theoretical Importance and Measurement Validity. Journal of Happiness Studies, 16(1), 211-239. https://doi.org/10.1007/s10902-014-9505-8

Hou, XL, Wang, HZ, Hu, TQ, Gentile, DA, Gaskin, J, \& Wang, JL (2019). The relationship between perceived stress and problematic social networking site use among Chinese college students. Journal $\begin{array}{lll}\text { of Behavioral } \quad \text { Addictions, } & \text { 306-317. }\end{array}$ https://doi.org/10.1556/2006.8.2019.26

Humphrey, KR (2013). Using a Student-Led Support Group to Reduce Stress and Burnout Among BSW Students. Social Work $\begin{array}{lll}\text { with } \quad \text { Groups, } & \text { 36(1), }\end{array}$ https://doi.org/10.1080/01609513.2012.712905

Ivanova, A, Gorbaniuk, O, Błachnio, A, Przepiórka, A, Mraka, N, Polishchuk, V, \& Gorbaniuk, J (2020). Mobile Phone Addiction, Phubbing, and Depression Among Men and Women: A Moderated Mediation Analysis. Psychiatric Quarterly, 91(3), 655-668. https://doi.org/10.1007/s11126-020-09723-8

James, D, \& Drennan, J (2005). Exploring Addictive Consumption of Mobile Phone Technology. In ANZMAC 2005 Conference: Electronic Marketing (Ed.), Exploring Addictive Consumption of Mobile Phone Technology. ANZMAC.

Kaparounakia Chrysi, K, Patsalia, ME, Mousaa, DP, Papadopouloua, EVK, Papadopouloua, KKK, \& Fountoulakis, KN (2020). University students' mental health amidst the COVID-19 quarantine in Greece. Psychiatry Research Journal, January.

Kato, TA, Katsuki, R, Kubo, H, Shimokawa, N, Sato-Kasai, M, Hayakawa, K, Kuwano, N, Umene-Nakano, W, Tateno, M, Setoyama, D, Kang, D, Watabe, M, Sakamoto, S, Teo, AR, \& Kanba, S (2019). Development and validation of the 22-item Tarumi's Modern-Type Depression Trait Scale: Avoidance of Social Roles, Complaint, and Low Self-Esteem (TACS-22). Psychiatry and Clinical Neurosciences, 73(8), 448-457. https://doi.org/10.1111/pcn.12842

Kitazawa, M, Yoshimura, M, Hitokoto, H, Sato-Fujimoto, Y, Murata, M, Negishi, K, Mimura, M, Tsubota, K, \& Kishimoto, T (2019). Survey of the effects of internet usage on the happiness of Japanese university students. In Health and Quality of Life Outcomes, 17(1). BioMed Central Ltd. https://doi.org/10.1186/s12955-019-1227-5

Kolhar, M, Kazi, RNA, \& Alameen, A (2021). Effect of social media use on learning, social interactions, and sleep duration among university students. Saudi Journal of Biological Sciences, 28(4), 2216-2222. https://doi.org/10.1016/j.sjbs.2021.01.010

Kramer, R (2021). Social networks, depression, and stress. In Handbook of research methods in health psychology. (pp. 170183). Routledge/Taylor \& Francis Group. https://doi.org/10.4324/9780429488320-16

Kroenke, K, Spitzer, RL, \& Williams, JBW (2001). The PHQ-9. Journal of General Internal Medicine, 16(9), 606-613. https://doi.org/10.1046/j.1525-1497.2001.016009606.x

Kwon, M, Kim, DJ, Cho, H, \& Yang, S (2014). The Smartphone Addiction Scale: Development and Validation of a Short Version for Adolescents. PLOS ONE, 8(12). https://doi.org/10.1371/journal.pone.0083558 
Lauckner, C, Hill, M, \& Ingram, LA (2020). An Exploratory Study of the Relationship between Social Technology Use and Depression among College Students. Journal of College Student Psychotherapy, 34(1), 33-39. https://doi.org/10.1080/87568225.2018.1508396

Leung, ANM, Law, W, Liang, YY, Au, ACL, Li, C, \& Ng, HKS (2021). What explains the association between usage of social networking sites (Sns) and depression symptoms? the mediating roles of selfesteem and fear of missing out. International Journal of Environmental Research and Public Health, 18(8). https://doi.org/10.3390/ijerph18083916

Li, LZ, Bian, JY, \& Wang, S (2021). Moving beyond family: unequal burden across mental health patients' social networks. Quality of Life Research, 30(7), 1873-1879. https://doi.org/10.1007/s11136021-02782-9

Lin, L, Sidani, JE, Shensa, A, Radovic, A, Miller, E, Colditz, JB, Hoffman, BL, Giles, LM, \& Primack, BA (2016). ASSOCIATION BETWEEN SOCIAL MEDIA USE AND DEPRESSION AMONGU.S. YOUNG ADULTS. Depression and Anxiety, 33(4), 323-331. https://doi.org/10.1002/da.22466

Mancini, MN (2019). Development and Validation of the Secondary Traumatic Stress Scale in a Sample of Social Media Users. Thesis, Cleveland State University.

Marino, C, Gini, G, Vieno, A, \& Spada, MM (2018). The associations between problematic Facebook use, psychological distress and well-being among adolescents and young adults: A systematic review and meta-analysis. Journal of Affective Disorders, 226, 274281. https://doi.org/10.1016/j.jad.2017.10.007

Martínez-Líbano, J (2020). Salud mental en estudiantes chilenos durante confinamiento por Covid-19: revisión bibliográfica Mental. Revista Educación Las Américas, 2 https://doi.org/https://doi.org/10.35811/rea.v10i2.126

Martínez-Líbano, J, \& Yeomans Cabrera, MM (2021). SUICIDAL IDEATION AND SUICIDAL THOUGHTS IN UNIVERSITY STUDENTS DURING THE COVID-19 PANDEMIC: A SYSTEMATIC REVIEW. Revista Argentina de Clinica Psicologica, 30(2), 390-405. https://doi.org/10.24205/03276716.2020.40

Martínez-Líbano, J, Yeomans Cabrera, MM, González Campusano, N, \& Campos Flores, E (2021). Emotional Exhaustion and Mental Health in a Sample of Chilean Social Science Students During the COVID-19 Pandemic. \{PSOCIAL\} Journal of Research in Social Psychology, 7(2), 69-81.

Mason, MJ, Schmidt, C, Abraham, A, Walker, L, \& Tercyak, K (2009). Adolescents' Social Environment and Depression: Social Networks, Extracurricular Activity, and Family Relationship Influences. Journal of Clinical Psychology in Medical Settings, 16(4), 346-354. https://doi.org/10.1007/s10880-009-9169-4

Maulik, PK, Eaton, WW, \& Bradshaw, CP (2010). The effect of social networks and social support on common mental disorders following specific life events. Acta Psychiatrica Scandinavica, 122(2), 118128. https://doi.org/10.1111/j.1600-0447.2009.1511.x

Moher, D, Liberati, A, Tetzlaff, J, Altman, DG, \& Group, TP (2009). Preferred Reporting Items for Systematic Reviews and MetaAnalyses: The PRISMA Statement. PLOS Med, 6(7). https://doi.org/10.1371/journal.pmed.1000097
Moreno Vallejo, B (2020). Autoestima en la Actualidad: ¿Cómo afectan las Redes Sociales?. Tesis, Universidad Pontificia de Madrid.

Moromizato, MS, Ferreira, DBB, Souza, LSM, Leite, RF, Macedo, FN, \& Pimentel, D (2017). O Uso de Internet e Redes Sociais e a Relação com Indícios de Ansiedade e Depressão em Estudantes de Medicina. Revista Brasileira de Educação Médica, 41(4), 497504. https://doi.org/10.1590/1981-52712015v41n4rb20160118

Newman, L, Stoner, C, \& Spector, A (2021). Social networking sites and the experience of older adult users: a systematic review. Ageing and Society, $\quad 41(2)$, $377-402$. https://doi.org/10.1017/S0144686X19001144

Osada, H (2013). Internet addiction in Japanese college students: Is Japanese version of Internet Addiction Test (JIAT) useful as a screening tool. Bulletin of Senshu University School of Human Sciences, 3(1), 71-80.

Oyanedel, JC, \& Paez, D (2021). Editorial: Social Belongingness and Well-Being: International Perspectives. Frontiers in Psychology, 12, 735507. https://doi.org/10.3389/fpsyg.2021.735507

Padilla-Romero, C, \& Ortega-Blas, J (2017). Adicción a las redes sociales y sintomatología depresiva en universitarios. CASUS. Revista De Investigación Y Casos En Salud, 2, 47-53. https://doi.org/10.35626/casus.1.2017.31

Page, M, McKenzie, J, Bossuyt, P, Boutron, I, Hoffmann, T, Mulrow, C, Shamseer, L, Tetzlaff, J, \& Moher, D (2020). Updating guidance for reporting systematic reviews: development of the PRISMA 2020 statement. September. https://doi.org/10.31222/osf.io/jb4dx

Pantic, I (2014). Online Social Networking and Mental Health. Cyberpsychology, Behavior, and Social Networking, 17(10), 652657. https://doi.org/10.1089/cyber.2014.0070

Przybylski, AK, Murayama, K, DeHaan, CR, \& Gladwell, V (2013). Motivational, emotional, and behavioral correlates of fear of missing out. Computers in Human Behavior, 29(4), 1841-1848. https://doi.org/10.1016/j.chb.2013.02.014

Qudah, MFA, Albursan, IS, Bakhiet, SFA, Hassan, EMAH, Alfnan, AA, Aljomaa, SS, \& AL-khadher, MMA (2019). Smartphone Addiction and Its Relationship with Cyberbullying Among University Students. International Journal of Mental Health and Addiction, 17(3), 628-643. https://doi.org/10.1007/s11469-018-0013-7

Radloff, LS (1977). The CES-D Scale: A Self-Report Depression Scale for Research in the General Population. Applied Psychological Measurement, 1(3), 385-401. https://doi.org/10.1177/014662167700100306

Ragheb, W, Aze, J, Bringay, S, \& Servajean, M (2021). Negatively Correlated Noisy Learners for At-risk User Detection on Social Networks: A Study on Depression, Anorexia, Self-harm and Suicide. IEEE Transactions on Knowledge and Data Engineering, 1. https://doi.org/10.1109/TKDE.2021.3078898

Ramírez-Gil, E, Cuaya-Itzcoatl, IG, Guzmán-Pimentel, M, RojasSolís, JL, Ramírez-Gil, E, Cuaya-Itzcoatl, IG, Guzmán-Pimentel, M, \& Rojas-Solís, JL (2021). Adicción a las redes sociales y procrastinación académica en universitarios durante el confinamiento por COVID-19. Dilemas Contemporáneos: Educación, Política y Valores, 8(SPE4). https://doi.org/10.46377/dilemas.v8i.2771 
Rasmussen, EE, Punyanunt-Carter, N, LaFreniere, JR, Norman, MS, \& Kimball, TG (2020). The serially mediated relationship between emerging adults' social media use and mental well-being. Computers in Human Behavior, 102, 206-213. https://doi.org/10.1016/j.chb.2019.08.019

Rosenberg, M (1965). Rosenberg Self-Esteem Scale. APA PsycTests.

Rusca, R, Onwuchekwa, IF, Kinane, C, \& Maclnnes, D (2021). Comparing the social networks of service users with long term mental health needs living in community with those in a general adult in-patient unit. International Journal of Social Psychiatry, 00207640211017590. https://doi.org/10.1177/00207640211017590

Sadri, AM, Hasan, S, \& Ukkusuri, S (2019). Joint inference of user community and interest patterns in social interaction networks. Social Network Analysis and Mining, 9(1), 11. https://doi.org/10.1007/s13278-019-0551-4

Saini, N, Sangwan, G, Verma, M, Kohli, A, Kaur, M, \& Lakshmi, PVM (2020). Effect of Social Networking Sites on the Quality of Life of College Students: A Cross-Sectional Study from a City in North India. Scientific World Journal, 2020. https://doi.org/10.1155/2020/8576023

Sharif Hossen, M, \& Aminul Islam, M (2021). Understanding and Analyzing Social Network Structure Among University Students. https://doi.org/10.21203/rs.3.rs-705159/v1

Smyth, N, Siriwardhana, C, Hotopf, M, \& Hatch, SL (2015). Social networks, social support, and psychiatric symptoms: social determinants and associations within a multicultural community population. Social Psychiatry and Psychiatric Epidemiology, 50(7), 1111-1120. https://doi.org/10.1007/s00127-014-0943-8

Spielberger, CD, Gorsuch, RL, \& Lushene, RE (2015). STAI, cuestionario de ansiedad estado-rasgo. Manual. TEA.

Spitzer, RL, Kroenke, K, Williams, JBW, \& Löwe, B (2006). A Brief Measure for Assessing Generalized Anxiety Disorder: The GAD-7. Archives of Internal Medicine, 166(10), 1092-1097. https://doi.org/10.1001/archinte.166.10.1092

Tasnim, R, Islam, S, Hossain, S, Sikder, T, \& Potenza, MN (2020). Children and Youth Services Review Suicidal ideation among Bangladeshi university students early during the COVID-19 pandemic: Prevalence estimates and correlates. Children and Youth Services Review, 119(September), 105703. https://doi.org/10.1016/j.childyouth.2020.105703

Tateno, M, Kim, DJ, Teo, AR, Skokauskas, N, Guerrero, APS, \& Kato, TA (2018). Smartphone addiction in Japanese college students: Usefulness of the japanese version of the smartphone addiction scale as a screening tool for a new form of internet addiction. Psychiatry Investigation, 16(2), 115-120. https://doi.org/10.30773/pi.2018.12.25.2

Tateno, M, Teo, AR, Ukai, W, Kanazawa, J, Katsuki, R, Kubo, H, \& Kato, TA (2019). Internet addiction, smartphone addiction, and hikikomori trait in Japanese young adult: Social isolation and social network. Frontiers in Psychiatry, 10(JULY). https://doi.org/10.3389/fpsyt.2019.00455

Teo, AR, Chen, Jl, Kubo, H, Katsuki, R, Sato-Kasai, M, Shimokawa, N. Hayakawa, K, Umene-Nakano, W, Aikens, JE, Kanba, S, \& Kato, TA (2018). Development and validation of the 25 -item Hikikomori Questionnaire (HQ-25). Psychiatry and Clinical Neurosciences, 72(10), 780-788. https://doi.org/10.1111/pcn.12691
Thomée, S, Härenstam, A, \& Hagberg, M (2011). Mobile phone use and stress, sleep disturbances, and symptoms of depression among young adults - a prospective cohort study. BMC Public Health, 11(1), 66. https://doi.org/10.1186/1471-2458-11-66

Urrútia, G y Bonfill, X (2013). La declaración prisma: un paso adelante en la mejora de las publicaciones de la revista española de salud pública. Rev Esp Salud Pública, 99-102.

Vergara, L (2020). Imagen corporal y redes sociales en jóvenes de sexo femenino en etapa de adolescencia temprana. Tesis, Universidad de Chile. http://repositorio.uchile.cl/handle/2250/176181

Villacampa, J, Ingram, GPD, Martí-Vilar, M, \& Olivera-La Rosa, A (2018). An investigation of Facebook users' implicit associations between Facebook, sexual and prosocial behavior. Heliyon, 4(9). https://doi.org/10.1016/j.heliyon.2018.e00811

Wang, HZ, Yang, TT, \& Gaskin, J (2019). THE LONGITUDINAL ASSOCIATION BETWEEN PASSIVE SOCIAL NETWORKING SITE USAGE AND DEPRESSIVE SYMPTOMS: THE MEDIATING ROLE OF ENVY AND MODERATING ROLE OF LIFE SATISFACTION. Journal of Social and Clinical Psychology, 38(3).

Watson, D, Clark, LA, \& Tellegen, A (1988). Development and validation of brief measures of positive and negative affect: The PANAS scales. JOURNAL OF PERSONALITY AND SOCIAL PSYCHOLOGY, 54(6), 1063-1070. https://doi.org/10.1037/00223514.54.6.1063

Wojdan, W, Wdowiak, K, Witas, A, Drogoń, J, \& Brakowiecki, W (2021). The impact of social media on the lifestyle of young people. Polish Journal of Public Health, 130(1), 8-13. https://doi.org/10.2478/piph-2020-0003

Xin, M, Luo, S, She, R, Yu, Y, Wang, S, Lin, D, Wang, H, Wang, Z, \& Lau, JT (2020). Negative Cognitive and Psychological Correlates of Mandatory Quarantine During the Initial COVID-19 Outbreak in China. American Psychologist, 75(5), 607-617.

Yana Salluca, M, Sucari Turpo, WG, Adco Valeriano, H, Alanoca Gutierrez, R, \& Yana Salluca, N (2019). FOMO syndrome and degeneration of written language in elementary school students. Comuni@cción: Revista de Investigación En Comunicación y Desarrollo, 10(2), 131-139. https://doi.org/10.33595/22261478.10.2.385

Yang, D, Tu, C, \& Dai, X (2020). The Effect of the 2019 Novel Coronavirus Pandemic on College Students in Wuhan. Psychological Trauma: Theory, Research, Practice, and Policy, 12, 6-14.

Yeomans, MM, \& Silva, A (2020). Pedagogical and Psychosocial Implications of Quarantine by Covid-19 on Chilean Students. Revista Educación Las Américas, 10.

Young, K (2016). Internet addiction test (IAT). In Stoelting.

Zhang, XX, Rost, DH, Wang, JL, \& Reynolds, KJ (2020). ACTIVE AND PASSIVE SOCIAL NETWORKING SITES USAGE AND NEGATIVE EMOTIONS: A RECIPROCAL RELATIONSHIP? In Journal of Social and Clinical Psychology, 39(3).

Zhao, N, \& Zhou, G (2020). Social Media Use and Mental Health during the COVID-19 Pandemic: Moderator Role of Disaster Stressor and Mediator Role of Negative Affect. Applied Psychology: Health and Well-Being, 12(4), 1019-1038. https://doi.org/https://doi.org/10.1111/aphw.12226 
Zhou, TH, Hu, GL, \& Wang, L (2019). Psychological disorder identifying method based on emotion perception over social networks. International Journal of Environmental Research and Public Health, 16(6). https://doi.org/10.3390/ijerph16060953 